Universidad Nacional Experimental Francisco de Miranda (UNEFM). Santa Ana de Coro. Venezuela

Priscila Nataly Chicaiza-Morocho; Darwin Gabriel García-Herrera; Sandra Elizabeth Mena-Clerque Juan Carlos Erazo-Álvarez

DOI $10.35381 / \mathrm{cm} . v 6 \mathrm{i} 3.397$

\title{
WhatsApp como estrategias para fortalecer el trabajo colaborativo
}

\section{WhatsApp with strategies to strengthen collaborative}

\author{
Priscila Nataly Chicaiza-Morocho \\ priscila.chicaiza.74@est.ucacue.edu.ec \\ Universidad Católica de Cuenca, Azogues \\ Ecuador \\ https://orcid.org/0000-0002-6907-0889 \\ Darwin Gabriel García-Herrera \\ dggarciah@ucacue.edu.ec \\ Universidad Católica de Cuenca, Azogues \\ Ecuador \\ https://orcid.org/0000-0001-6813-8100 \\ Sandra Elizabeth Mena-Clerque \\ sandramena@ucacue.edu.ec \\ Universidad Católica de Cuenca, Cuenca \\ Ecuador \\ https://orcid.org/0000-0002-9186-2161 \\ Juan Carlos Erazo-Álvarez \\ jcerazo@ucacue.edu.ec \\ Universidad Católica de Cuenca, Cuenca \\ Ecuador \\ https://orcid.org/0000-0001-6480-2270
}

Recibido: 20 de agosto de 2020

Aprobado: 15 de noviembre de 2020 
Universidad Nacional Experimental Francisco de Miranda (UNEFM). Santa Ana de Coro. Venezuela

Priscila Nataly Chicaiza-Morocho; Darwin Gabriel García-Herrera; Sandra Elizabeth Mena-Clerque Juan Carlos Erazo-Álvarez

\title{
RESUMEN
}

El objetivo de la presente investigación es proponer estrategias que fortalezcan el trabajo colaborativo en los estudiantes de básica superior especialmente de zonas rurales con el uso del WhatsApp. Para ello se empleó una metodología descriptiva no experimental transversal, se aplicó encuesta a docentes del circuito 01D06 del cantón Paute. Con los resultados obtenidos se pudo evidenciar que los docentes están de acuerdo en usar el WhatsApp para fomentar el trabajo colaborativo, pero que es indispensable definir normativas para el uso adecuado del mismo. Es así que se realizó una propuesta enfocada en las siguientes estrategias: Trabajo grupal, elaboración de normativas e intercambio de opiniones, que direccionarán y fomentarán las directrices de un trabajo estudiantil dinámico, interactivo, equitativo y tecnológico.

Descriptores: Medios sociales; tecnología de la información; tecnología educacional. (Palabras tomadas del tesauro UNESCO).

\begin{abstract}
The objective of this research is to propose strategies that strengthen collaborative work in upper secondary students, especially in rural areas, with the use of WhatsApp. For this, a descriptive, non-experimental cross-sectional methodology was used, a survey was applied to teachers of the 01D06 circuit of the Paute canton. With the results obtained, it was possible to show that the teachers agree to use WhatsApp to promote collaborative work, but that it is essential to define regulations for its proper use. Thus, a proposal was made focused on the following strategies: Group work, development of regulations and exchange of opinions, which will direct and promote the guidelines of dynamic, interactive, equitable and technological student work.
\end{abstract}

Descriptors: Social media; information technology; educational technology. (Words taken from the UNESCO thesaurus). 
Universidad Nacional Experimental Francisco de Miranda (UNEFM). Santa Ana de Coro. Venezuela

Priscila Nataly Chicaiza-Morocho; Darwin Gabriel García-Herrera; Sandra Elizabeth Mena-Clerque Juan Carlos Erazo-Álvarez

\section{INTRODUCCIÓN}

La educación utiliza diversas estrategias en el proceso de enseñanza y aprendizaje, donde el docente crea ambientes interactivos entre estudiantes, sumado a ello, la tecnología ha dotado de varios recursos didácticos que dinamizan esta relación. El problema que surge con estas nuevas herramientas tecnológicas es su accesibilidad, ya que no todos quienes conforman la comunidad educativa cuentan con los recursos [computador o internet] que les permita hacer uso de las mismas y esto es más notorio en instituciones educativas rurales, y que decir de los planteles uni y pluridocentes, en donde las familias apenas cuentan con los servicios básicos, luz, y agua potable.

También, se debe considerar que los estudiantes de las zonas rurales no poseen habilidades en el manejo del computador, a pesar de ser nativos digitales, pues en sus hogares y escuelas no cuentan con este recurso, peor aún del servicio de internet fijo, impidiéndoles formar parte de esta nueva era tecnológica, privándolos de la oportunidad de aplicar un aprendizaje conectivista.

Ante esta situación, el docente se ve en la necesidad de buscar diversas maneras de implementar estrategias tecnológicas, y una de las opciones más viables es a través del uso del celular, pues la mayoría de personas cuentan con este dispositivo, no por vanidad sino por la necesidad de comunicarse. Los dispositivos móviles actuales, cuentan con diversas Apps, sobre todo de redes sociales -Facebook, Instagram, WhatsApp, que en su mayoría sus descargas y uso son de manera gratuita, pues vienen incluidos en los planes móviles que se contratan, siendo WhatsApp el de mayor accesibilidad y fácil manejo (Rubio \& Perlado, 2015). WhatsApp, es una de las redes sociales más usadas en la actualidad y que fundamentada pedagógicamente y aplicada de manera correcta, puede brindar grandes beneficios a la calidad educativa, pues nos ayudará en gran medida a fortalecer la relación entre estudiantes y el docente (Tezer, Taşpolat, Sami , \& Fatih , 2017). 
Universidad Nacional Experimental Francisco de Miranda (UNEFM). Santa Ana de Coro. Venezuela

Priscila Nataly Chicaiza-Morocho; Darwin Gabriel García-Herrera; Sandra Elizabeth Mena-Clerque Juan Carlos Erazo-Álvarez

Es así que brindar a la sociedad una nueva visión del uso del WhatsApp, ya no únicamente como una red social, sino como una red didáctica de aprendizaje, a la que todos tienen acceso. Con ello, se logra que todos los estudiantes de la básica superior, usen las nuevas tecnologías, brindándoles la oportunidad de crear espacios de colaboración e interacción, cumpliendo a cabalidad el perfil de salida propuesto por el Ministerio de Educación, fortaleciendo valores que nos impulsen a aplicar correctamente la justicia, generando un espíritu solidario, capaz de promover la innovación respetando la cultura y dotando de una conciencia social que genere un cambio en la sociedad a través de una sana convivencia.

Por ello el presente artículo está enfocado al uso del WhatsApp, destacando la incógnita ¿Cómo puede contribuir el WhatsApp en el aprendizaje? El objetivo principal es proponer el uso del WhatsApp como una estrategia de aprendizaje que fortalece el trabajo colaborativo especialmente en estudiantes de zonas rurales, debido a que sus aprendizajes se han regido por mucho tiempo de manera tradicional y autónomo sin uso de recursos tecnológicos.

\section{Referencial teórico}

Con lo mencionado anteriormente surge la importancia de retomar aportes de diferentes autores que han tomado al WhatsApp como una herramienta para la educación, a partir de eso, (Suárez, 2017) se enfocó en la participación de varios alumnos españoles, quienes crearon grupos usando el WhatsApp, incluyendo al docente, para que pueda resolver inquietudes de la asignatura. Las herramientas que le permitió recopilar la información fueron: la entrevista y el cuestionario. Logrando que los estudiantes tengan una mayor interactividad, mejorando sus trabajos, haciendo de la tutoría, un factor clave en este proceso, logrando el desarrolló potencialidades en sus alumnos, así como también la creación de ambientes dinámicos y productivos. Dicho de otra manera, el WhatsApp es un apoyo para las tutorías académicas. 
Universidad Nacional Experimental Francisco de Miranda (UNEFM). Santa Ana de Coro. Venezuela

Priscila Nataly Chicaiza-Morocho; Darwin Gabriel García-Herrera; Sandra Elizabeth Mena-Clerque Juan Carlos Erazo-Álvarez

Así mismo (Benítez \& Marquina , 2018) quienes crearon dos grupos de estudiantes para su investigación a través del aula virtual y el grupo de WhatsApp, debido a que los estudiantes provenían de diferentes ciudades de Venezuela. Estos autores recolectaron la información en base a la forma de interacción entre docentes y estudiantes. Es así como llegan a la conclusión que los grupos de discusión creados en WhatsApp permiten un acompañamiento y apoyo de las actividades de enseñanza y aprendizaje y más aún de espacios colaborativos en donde los estudiantes trabajan para lograr una meta en común.

Con el estudio de (Ruvalcaba, Torres, Carmona, \& Pérez, 2019), se menciona que tanto el WhatsApp como el Facebook son utilizados con mayor frecuencia dentro de clases en ciertas áreas de estudio [económico-administrativo], en cambio en otras especialidades [químico-biólogo] usan para los diálogos entre amigos y entretenimiento, en definitiva estos medios de comunicación instantáneos dotan al estudiantado de múltiples funciones aplicables en el medio social como en el educativo.

Por su parte (Rodríguez, 2020), en base a la investigación en Costa Rica entre los años 2018 y 2019, en donde experimentó el uso del WhatsApp en 7 cursos diferentes. Diseñó y aplicó estrategias didácticas con el uso de la App en la enseñanza- aprendizaje. Al finalizar el ciclo aplicó un cuestionario en línea para los estudiantes, que le permitió conocer las experiencias que obtuvieron durante las clases, manifestando que es una herramienta útil y de apoyo para el proceso de enseñanza, especialmente para niveles universitarios.

Concluyendo con (Zulkanain, Miskon, \& Syed, 2020) quienes enfocan al WhatsApp desde una perspectiva educativa, mediante un marco de comunidad de investigación con la ayuda de expertos quienes aplicaron el marco pedagógico propuesto, se demostró el trabajo positivo de la app en el aprendizaje, ya sea en grupos o de forma individual, además consideraron que se puede percibir aspectos emocionales positivos y negativos como: felicidad, entusiasmos, espontaneidad, timidez, inseguridad, comunicación pasiva, 
Universidad Nacional Experimental Francisco de Miranda (UNEFM). Santa Ana de Coro. Venezuela

Priscila Nataly Chicaiza-Morocho; Darwin Gabriel García-Herrera; Sandra Elizabeth Mena-Clerque Juan Carlos Erazo-Álvarez

etc. En definitiva, el uso de este medio es útil para el trabajar con los aspectos mencionados anteriormente, fomentando valores de respeto y colaboración para un aprendizaje fructífero en ambientes armónicos.

\section{TIC en la Educación}

Las tecnologías de información y comunicación [TIC], según (Quispe, 2019) ha facilitado la forma de vida y la transferencia de saberes dentro de un entorno global llamado conectivismo, aprendizaje influenciada por la implementación de herramientas digitales en el campo educativo (Siemens, 2004).

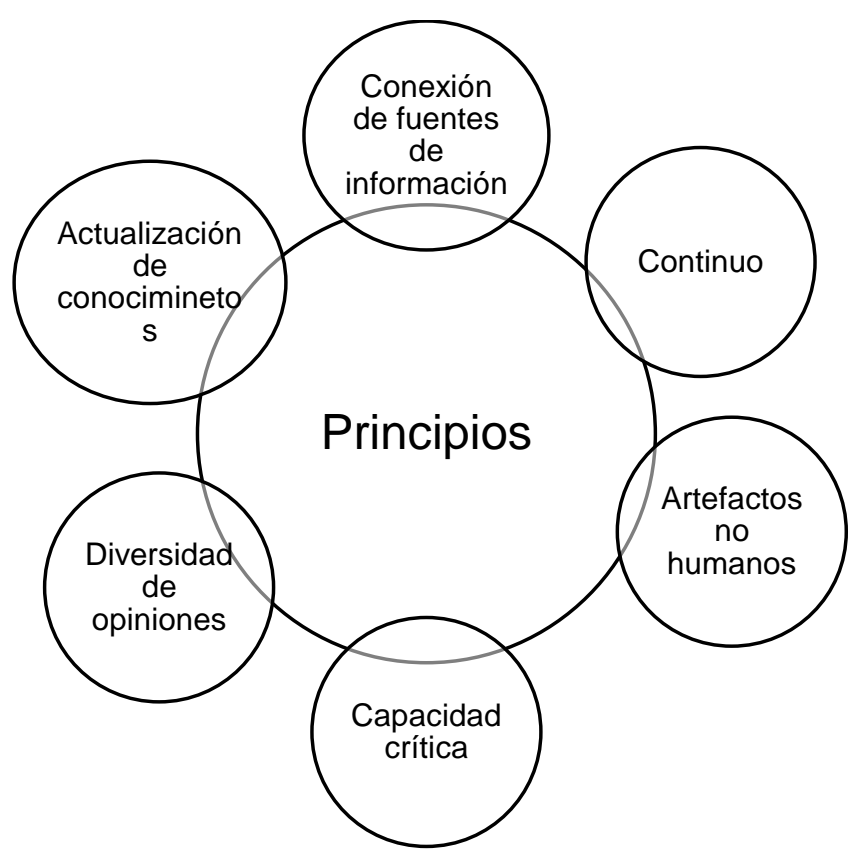

Figura 1. Determinación de los principios del conectivismo.

Fuente: Adaptado de Siemens, (2004). 
Universidad Nacional Experimental Francisco de Miranda (UNEFM). Santa Ana de Coro. Venezuela

Priscila Nataly Chicaiza-Morocho; Darwin Gabriel García-Herrera; Sandra Elizabeth Mena-Clerque Juan Carlos Erazo-Álvarez

El conectivismo según sus principios permite activar, crecer y evolucionar el conocimiento, no de una manera interna ni individualista sino de un modo universal, siendo necesarias nuevas herramientas que proporcionen información, donde los alumnos progresen en esta era digital.

El Internet, es una de las innovaciones tecnológicas que se involucra en el ámbito educativo, debido a que permite la propagación de información y la apertura de comunidades virtuales. Es así que esta herramienta nos mantiene en comunicación permanente desde diversos enfoques, ya sean estos sociales, personales y académicos. En el proceso de enseñanza - aprendizaje las tecnologías de la información y comunicación destacan por ser los recursos de apoyo que el docente usa, pero no de manera continua, por lo que es indispensable aplicar dichos medios dentro y fuera del salón de clases.

\section{Estrategias de aprendizaje}

Durante la enseñanza de las diferentes asignaturas, el docente se ha dedicado a dictar contenidos teóricos, que con lleva a la memorización, con el único propósito de adquirir una calificación, haciendo que el aprendizaje sea fugaz. Las estrategias de aprendizaje más sobresalientes son: el aprendizaje basado en proyectos [ABP], el aprendizaje colaborativo $[A C]$, estudio de casos, redes colaborativas digitales, entre otros (Colorado \& Gutiérrez, 2016).

Toda estrategia de aprendizaje, para que sea óptima, debe garantizar un trabajo activo, logrando que el estudiante asimile nuevos conocimientos de forma participativa, convirtiéndolo en el centro del aprendizaje, potencializando sus habilidades, destrezas y actitudes en la solución de problemas, y dotándolos de comunicación interactiva que fortalece el trabajo colaborativo. Considerando lo expuesto, el WhatsApp, es la estrategia ideal para lograr un cambio radical en la educación, pues permite alcanzar todos los aspectos mencionados. 
Universidad Nacional Experimental Francisco de Miranda (UNEFM). Santa Ana de Coro. Venezuela

Priscila Nataly Chicaiza-Morocho; Darwin Gabriel García-Herrera; Sandra Elizabeth Mena-Clerque Juan Carlos Erazo-Álvarez

\section{Redes sociales: WhatsApp en el aprendizaje}

La era digital dio paso a las redes sociales, causando gran impacto en la sociedad. Es así que se define a la red social como una estructura de conexión entre varias personas mediante el servicio de internet, cuya finalidad es obtener una interacción activa y dinámica. Las redes sociales son un ecosistema, donde existe una variedad de servicios útiles para los usuarios, su función más sobresaliente es la comunicación, sea esta sincrónica o asincrónica.

Las redes sociales de acuerdo a (Marañón, 2012), pueden ser de carácter personal [Facebook, WhatsApp]; profesionales [Womenalia, Universia, Viadeo]; temáticas [cuentatuviaje.net, musicmakesfriends.com], y de escala local [Skyrock, Xianoei, Tuenti, Hyves]. De la clasificación mencionada, es importante destacar las redes de carácter personal, ya que son las que permiten el intercambio de contenido multimedia [imágenes, videos, audios, enlaces, archivos] entre sus contactos. Las que se utilizan con mayor frecuencia son: Facebook, Twitter, Instagram y especialmente el WhatsApp, esta última, puede ser empleada como una estrategia didáctica que permita fortalecer el aprendizaje colaborativo dentro del ambiente educativo.

Por tal motivo es importante resaltar todo lo concerniente a WhatsApp, partiendo de su nombre en inglés: 'what's up' en español significa '¿qué pasa?' y app es abreviatura de la palabra aplicaciones. En definitiva, es una red social muy famosa en la actualidad, cuyo nombre es conocido a nivel mundial, debido a que es gratuita y de fácil manejo. Varios autores definen a esta app, es así que (Vilches, 2019) determina que el WhatsApp es una aplicación que se descarga a un móvil de manera gratuita y de fácil acceso; (Benítez \& Marquina , 2018) lo establecen como un medio de mensajería instantánea que al estar conectado en el internet se puede realizar llamadas, video llamadas y enviar/recibir mensajes multimedia [audio, imagen, video y documentos]. Es así como permite una comunicación sincrónica o asincrónica entre usuarios y la facilidad de adquirir cualquier tipo de información al instante. 
Universidad Nacional Experimental Francisco de Miranda (UNEFM). Santa Ana de Coro. Venezuela

Priscila Nataly Chicaiza-Morocho; Darwin Gabriel García-Herrera; Sandra Elizabeth Mena-Clerque Juan Carlos Erazo-Álvarez

WhatsApp se ha convertido en una aplicación de gran índole tanto para uso personal como educativo. En relación con ello (Rodríguez, 2020) destaca el aprendizaje móvil el cual crea un nivel diferente de conocimientos y espacios educativos distintos al paradigma acostumbrado. En otras palabras, esta aplicación es una herramienta muy útil para el aprendizaje, pues dinamiza el intercambio de información sin importar la distancia de quienes la usen, eliminando las barreras tradicionales de comunicación.

Además, (Casillas, Cabezas, \& Hernández, 2017) y (Gómez, 2017) consideran que el WhatsApp brinda a los dicentes expandir sus conocimientos sin estar encerrados en un salón de clase, permitiendo construir y compartir el conocimiento, a través de un aprendizaje colaborativo dando respuesta a las exigencias de la sociedad actual y, más concretamente, a las habilidades tecnológicas que deben poseer los estudiantes del siglo XXI. Es por eso que el docente debe estar en constante capacitación para poder contribuir y guiar a los estudiantes que pertenecientes a la generación z o centennials.

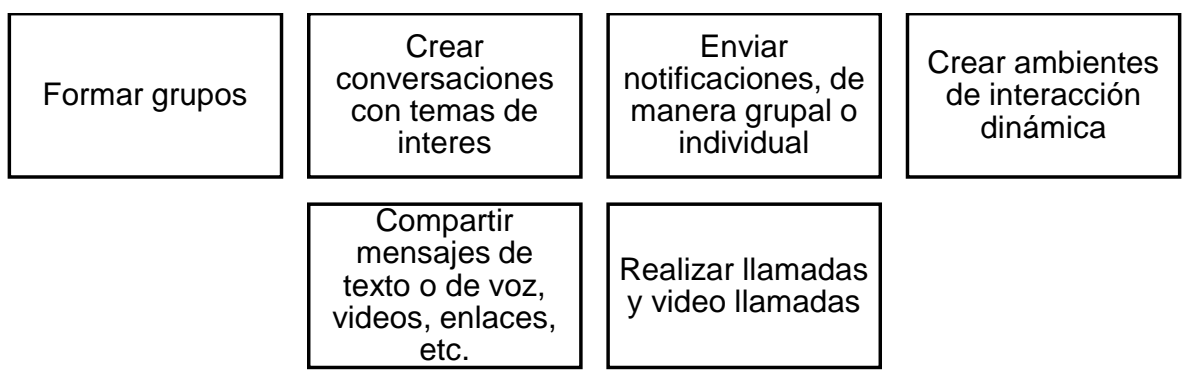

Figura 2. Actividades que el WhatsApp permite realizar. Fuente: Elaboración propia.

En definitiva, el WhatsApp tiene el potencial para ser empleado dentro del sistema educativo, pues permite realizar actividades que fortalecen en trabajo grupal de forma dinámica como se visualiza en la figura 2, que aportan significativamente al desarrollo de un aprendizaje colaborativo y cooperativo; mismo que se enfoca en potencializar un andamiaje grupal, basados en la responsabilidad, organización, motivación y solidaridad 
Universidad Nacional Experimental Francisco de Miranda (UNEFM). Santa Ana de Coro. Venezuela

Priscila Nataly Chicaiza-Morocho; Darwin Gabriel García-Herrera; Sandra Elizabeth Mena-Clerque Juan Carlos Erazo-Álvarez

al intercambiar o compartir conocimientos, ideas e información entre pares con un lenguaje común y con un mismo fin de aprender de uno mismo y de los demás ya sea en una aula o en casa.

Es así que esta aplicación puede ser empleada como estrategia de aprendizaje, pues permite realizar diversas actividades tales como: debate-foro, ya sea en pequeños grupos o toda el aula, donde expresan opiniones sobre el tema; lluvia de ideas, donde se pone en común todas las ideas o conocimientos, para establecer una conclusión o síntesis; controversia estructurada, dividir en grupo, asignar un tema, investigar el tema clasificando el contenido en aspectos positivos o negativos para luego ser debatido; preguntas y premios, dirigida a la participación de los estudiantes en busca de una solución al problema planteado por el docente y el grupo que mayor concordancia tienen en la solución es el triunfador (Delgado \& Solano, 2009).

\section{METODOLOGÍA}

La investigación presentada es de tipo descriptiva no experimental transversal, ya que se realizó en un solo tiempo, con un muestreo por conglomeración de 22 docentes de las diferentes instituciones del circuito 01D06 del cantón Paute. Para la recolección de datos se aplicó una encuesta mediante cuestionario de 18 preguntas en Microsoft Forms, la cual fueren aplicadas de manera online.

Se utilizo el programa IBM SPSS Statistics, para el análisis de datos cuantitativos y la respectiva validación de la encuesta, obteniendo una fiabilidad y coeficiente Alpha de Cronbach de 0.851 . Los datos obtenidos se encuentran analizados con un $95 \%$ de coeficiente de confianza, y en base a la prueba de normalidad de Shapiro-Willk los resultados evidencian que todas las variables son de tipo paramétrica es decir que $P$ es menor a 0.05 . 
Universidad Nacional Experimental Francisco de Miranda (UNEFM). Santa Ana de Coro. Venezuela

Priscila Nataly Chicaiza-Morocho; Darwin Gabriel García-Herrera; Sandra Elizabeth Mena-Clerque Juan Carlos Erazo-Álvarez

\section{RESULTADOS}

De las variables investigadas se analizo 8 de ellas, mismas que permiten responder al objetivo de investigacion, las cuales se muestran a contuciación:

\section{Tabla 1.}

Uso del WhatsApp como una estrategia de aprendizaje para fortalecer el trabajo colaborativo.

\begin{tabular}{cccccc}
\hline & & Frecuencia & Porcentaje & Porcentaje válido & $\begin{array}{c}\text { Porcentaje } \\
\text { acumulado }\end{array}$ \\
\hline \multirow{2}{*}{ Válidos } & Muy de acuerdo & 11 & 50,0 & 50,0 & 50,0 \\
& De acuerdo & 7 & 31,8 & 31,8 & 81,8 \\
& En desacuerdo & 3 & 13,6 & 13,6 & 95,5 \\
& Totalmente en desacuerdo & 1 & 4,5 & 4,5 & 100,0 \\
\cline { 2 - 6 } & \multicolumn{2}{c}{ Total } & 22 & 100,0 & 100,0
\end{tabular}

Fuente: Elaboración propia.

En la tabla 1, se puede visualizar que el $81,8 \%$ estan muy de acuerdo y de acuerdo del uso del WhatApp como una estrategia de aprendizaje, razón por la cual es importante establecer actividades que promueven y fortalezcan el trabajo colaborativo

Los resultados obtenidos sobre el uso del WhatApp en el nivel o subnivel de educación basica, se resume que el $86,4 \%$ de los docentes concuerdad que es mas factible trabajar esta App con los estudiantes de la basica superior, por ello es necesario proponer estategias para aplicar en las diferentes asignaturas, ver tabla 2. 
CIENCIAMATRIA

Revista Interdisciplinaria de Humanidades, Educación, Ciencia y Tecnología

Año VI. Vol. VI. N³. Edición Especial III. 2020

Hecho el depósito de ley: pp201602FA4721

ISSN-L: 2542-3029; ISSN: 2610-802X

Universidad Nacional Experimental Francisco de Miranda (UNEFM). Santa Ana de Coro. Venezuela

Priscila Nataly Chicaiza-Morocho; Darwin Gabriel García-Herrera; Sandra Elizabeth Mena-Clerque Juan Carlos Erazo-Álvarez

Tabla 2.

WhatsApp aplicable para los estudiantes.

\begin{tabular}{cccccc}
\hline & & Frecuencia & Porcentaje & Porcentaje válido & $\begin{array}{c}\text { Porcentaje } \\
\text { acumulado }\end{array}$ \\
\hline \multirow{3}{*}{ Válidos } & Básica superior & 19 & 86,4 & 86,4 & 86,4 \\
& Básica media & 3 & 13,6 & 13,6 & 100,0 \\
\cline { 2 - 6 } & Total & 22 & 100,0 & 100,0 & \\
\hline
\end{tabular}

Fuente: Elaboración propia

Tabla 3.

Relación en crear normas para el uso del WhatsApp y Promueve ambientes colaborativos y de interacción entre los estudiantes a través del WhatsApp.

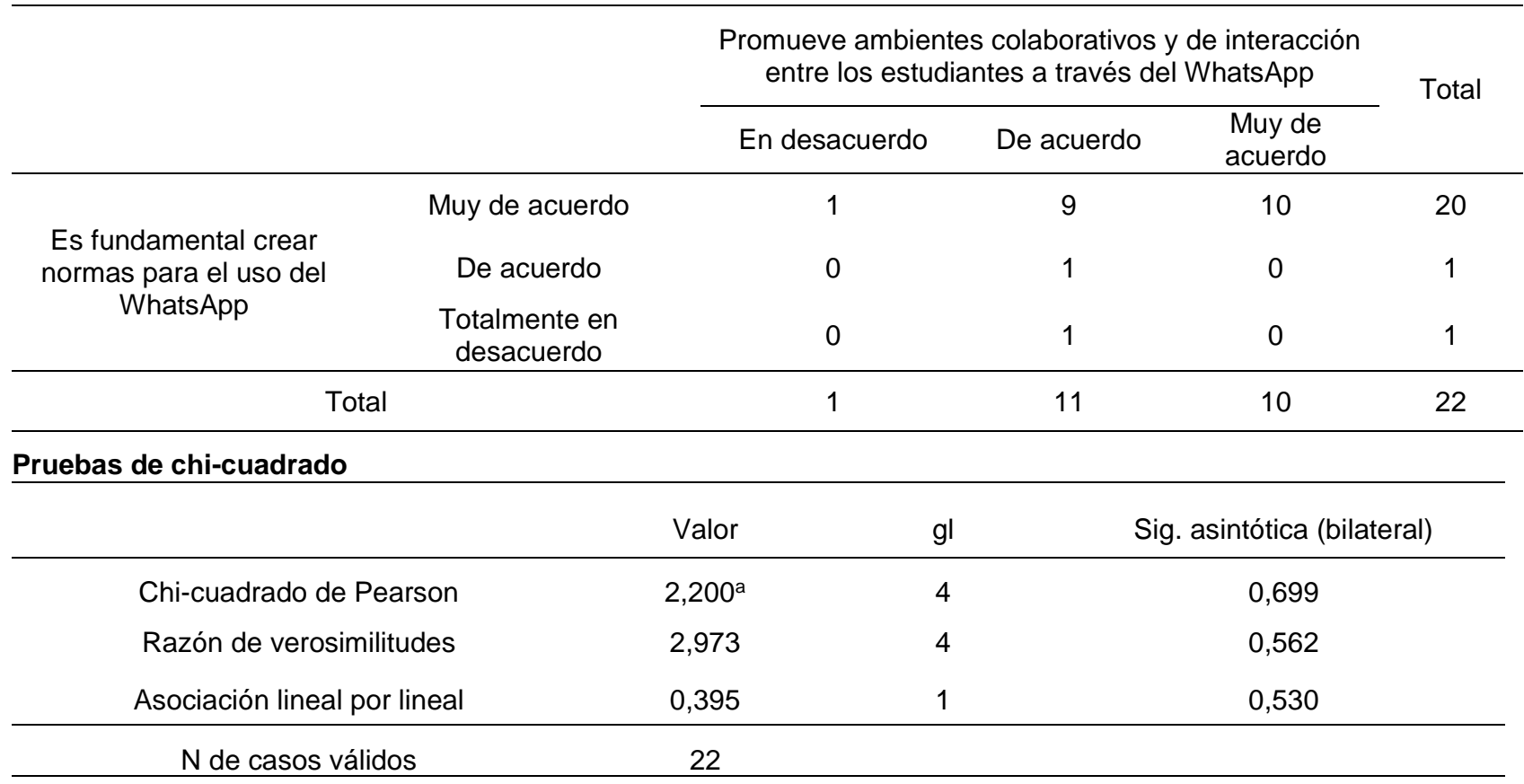

a. 7 casillas $(77,8 \%)$ tienen una frecuencia esperada inferior a 5 . La frecuencia mínima esperada es 0,05 .

Fuente: Elaboración propia. 
Universidad Nacional Experimental Francisco de Miranda (UNEFM). Santa Ana de Coro. Venezuela

Priscila Nataly Chicaiza-Morocho; Darwin Gabriel García-Herrera; Sandra Elizabeth Mena-Clerque Juan Carlos Erazo-Álvarez

La tabla 3 muestra el analisis de Chi-cuadrado, cuyo valor de P es 0,699, es decir mayor a 0,05, lo cual se asume una hipotesis nula, demostrando la no existe de relación entre la frecuencia, crear normas para el uso del WhatsApp y promueve ambientes colaborativos y de interacción entre los estudiantes a través del WhatsApp, datos que permiten afianzar la creación de normativas para promover ambientes interactivos y colaborativos entre los estudiantes.

Con respecto a la tabla 4,5,6 y 7 , las variables se encuentran enfocadas a las actividades que los docentes realizan a través del WhatsApp, siendo la tabla 4 y 5 actividades individuales y grupales la tabla 6 y 7 . Con referencia a la tabla 4 el 95,59\% siempre y casi siempre receptan los deberes y trabajos por el WhatsApp, en la tabla 5 el $100 \%$ de los docentes siempre y casi siempre utilizan el WhatsApp para enviar notificaciones a sus estudiantes, el 59,1\% nunca han utilizado el WhatsApp para realizar trabajos grupas, ver tabla 6 , en la tabla 7 con respecto al foro de discusión el $45,5 \%$ nunca y 27,3 pocas veces han realizado esta actividad. Desde esta perspectiva la mayoría de los docentes no han realizado actividades grupales donde se pueda evidenciar el trabajo colaborativo, por ende, se desea determinar las actividades específicas que ayuden a fortalecer el aprendizaje especialmente colaborativo.

\section{Tabla 4.}

Recepción de deberes y trabajos.

\begin{tabular}{|c|c|c|c|c|c|}
\hline & & Frecuencia & Porcentaje & Porcentaje válido & $\begin{array}{l}\text { Porcentaje } \\
\text { acumulado }\end{array}$ \\
\hline \multirow[t]{4}{*}{ Válidos } & Siempre & 20 & 90,9 & 90,9 & 90,9 \\
\hline & Casi siempre & 1 & 4,5 & 4,5 & 95,5 \\
\hline & Nunca & 1 & 4,5 & 4,5 & 100,0 \\
\hline & Total & 22 & 100,0 & 100,0 & \\
\hline
\end{tabular}

Fuente: Elaboración propia. 
CIENCIAMATRIA

Revista Interdisciplinaria de Humanidades, Educación, Ciencia y Tecnología

Año VI. Vol. VI. N³. Edición Especial III. 2020

Hecho el depósito de ley: pp201602FA4721

ISSN-L: 2542-3029; ISSN: 2610-802X

Universidad Nacional Experimental Francisco de Miranda (UNEFM). Santa Ana de Coro. Venezuela

Priscila Nataly Chicaiza-Morocho; Darwin Gabriel García-Herrera; Sandra Elizabeth Mena-Clerque Juan Carlos Erazo-Álvarez

\section{Tabla 5.}

Notificaciones a los estudiantes.

\begin{tabular}{|c|c|c|c|c|c|}
\hline & & Frecuencia & Porcentaje & Porcentaje válido & $\begin{array}{l}\text { Porcentaje } \\
\text { acumulado }\end{array}$ \\
\hline \multirow[t]{3}{*}{ Válidos } & Siempre & 21 & 95,5 & 95,5 & 95,5 \\
\hline & Casi siempre & 1 & 4,5 & 4,5 & 100,0 \\
\hline & Total & 22 & 100,0 & 100,0 & \\
\hline
\end{tabular}

Fuente: Elaboración propia

Tabla 6.

Trabajo grupal.

\begin{tabular}{cccccc}
\hline & Frecuencia & Porcentaje & Porcentaje válido & $\begin{array}{c}\text { Porcentaje } \\
\text { acumulado }\end{array}$ \\
\hline \multirow{7}{*}{ Válidos } & Siempre & 2 & 9,1 & 9,1 & 9,1 \\
& Casi siempre & 1 & 4,5 & 4,5 & 13,6 \\
& A veces & 3 & 13,6 & 13,6 & 27,3 \\
& Pocas veces & 3 & 13,6 & 13,6 & 40,9 \\
& Nunca & 13 & 59,1 & 59,1 & 100,0 \\
\cline { 2 - 6 } & Total & 22 & 100,0 & 100,0 & \\
\hline
\end{tabular}

Fuente: Elaboración propia

Tabla 7.

Foro de discusión.

\begin{tabular}{cccccc}
\hline & Frecuencia & Porcentaje & Porcentaje válido & $\begin{array}{c}\text { Porcentaje } \\
\text { acumulado }\end{array}$ \\
\hline \multirow{6}{*}{ Válidos } & Siempre & 1 & 4,5 & 4,5 & 4,5 \\
& Casi siempre & 1 & 4,5 & 4,5 & 9,1 \\
& A veces & 4 & 18,2 & 18,2 & 27,3 \\
& Pocas veces & 6 & 27,3 & 27,3 & 54,5 \\
& Nunca & 10 & 45,5 & 45,5 & 100,0 \\
\cline { 2 - 6 } & Total & 22 & 100,0 & 100,0 & \\
\hline
\end{tabular}

Fuente: Elaboración propia. 
Universidad Nacional Experimental Francisco de Miranda (UNEFM). Santa Ana de Coro. Venezuela

Priscila Nataly Chicaiza-Morocho; Darwin Gabriel García-Herrera; Sandra Elizabeth Mena-Clerque Juan Carlos Erazo-Álvarez

Considerando los estudios de (Rodríguez, 2020), sobre el WhatsApp como un apoyo para niveles universitarios, no se concuerda ya que los resultados obtenidos en la encuesta los docentes determinan que se puede aplicar en estudiantes de la básica superior, y tomando los estudios de (Suárez, 2017) quien menciona la participación fundamental del docente para dar seguimiento a los procesos de aprendizaje, se coincide con dicho estudio, dado que el docente es el guía y orientador, en cuanto a los aspectos emocionales se concuerda con (Zulkanain, Miskon, \& Syed, 2020), pues es fundamental el trabajo de las emociones, que conlleva a mejorar la autoestima y crear en los discentes mayor intervención sin temor a equivocarse.

\section{PROPUESTA}

Mediante la investigación realizada, se demuestra que los docentes usan el WhatsApp como una herramienta de comunicación y recepción de deberes, mas no como estrategia de aprendizaje interdisciplinaria, por tal motivo, se pretende dar a conocer las utilidades de esta App que facilitan la aplicación de estrategias que dinamizan el rol estudiantil, transformando en el verdadero protagonista de su aprendizaje.

El WhatsApp es una herramienta que permite crear diferentes canales de comunicación a través de mensajes de texto, mensajes de audio, llamadas y video llamadas. A demás, brinda otros beneficios que optimiza la participación activa de los estudiantes, tales como: la creación de grupos y subgrupos de trabajo y la difusión de información a través de la publicación de estados de manera dinámica, por consiguiente, se proponen 3 estrategias que serán útiles para fortalecer el trabajo colaborativo con el uso del WhatsApp, mismas que se describen en la figura 3. 
Universidad Nacional Experimental Francisco de Miranda (UNEFM). Santa Ana de Coro. Venezuela

Priscila Nataly Chicaiza-Morocho; Darwin Gabriel García-Herrera; Sandra Elizabeth Mena-Clerque Juan Carlos Erazo-Álvarez

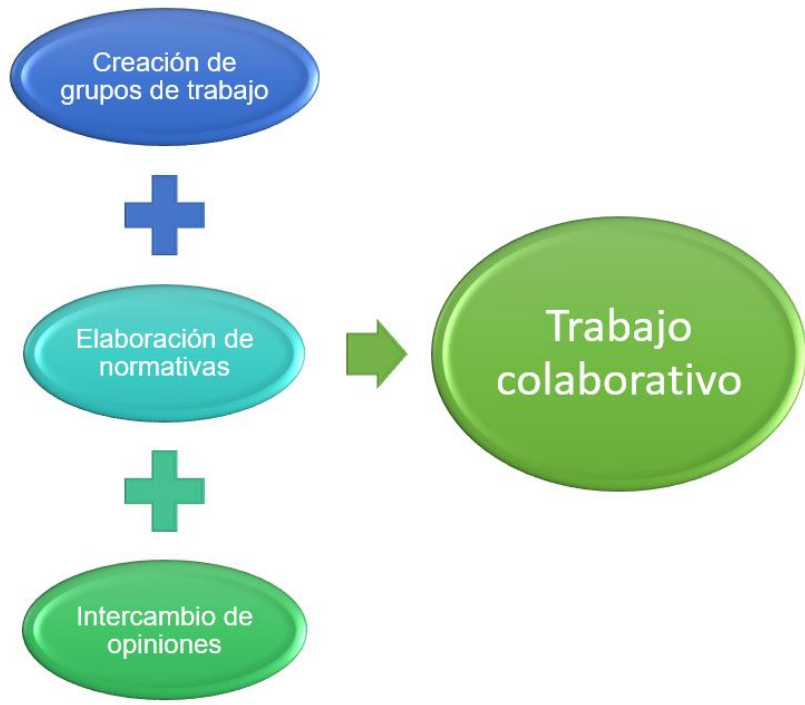

Figura 3. Estrategias para fortalecer el trabajo colaborativo con el uso del WhatsApp. Fuente: Elaboración propia.

Creación de grupos de trabajo: El WhatsApp nos brinda dos formas de crear grupos de trabajo: en la primera es indispensable tener registrado los contactos de las personas que integran el grupo, que se creará de acuerdo a la necesidad o asignatura de estudio como lo demuestra la figura 4 , en la segunda no es necesario contar con el registro total de contactos, pues basta con tener un solo de ellos, que permitirá crear el grupo de trabajo y posterior a ello generar un enlace de invitación que puede ser difundido a través de correo electrónico o página de Facebook ver figura 4 a las personas que deseemos que formen parte de dicho grupo. 


\section{CIENCIAMATRIA}

Revista Interdisciplinaria de Humanidades, Educación, Ciencia y Tecnología

Año VI. Vol. VI. N’3. Edición Especial III. 2020

Hecho el depósito de ley: pp201602FA4721

ISSN-L: 2542-3029; ISSN: 2610-802X

Universidad Nacional Experimental Francisco de Miranda (UNEFM). Santa Ana de Coro. Venezuela

Priscila Nataly Chicaiza-Morocho; Darwin Gabriel García-Herrera; Sandra Elizabeth Mena-Clerque Juan Carlos Erazo-Álvarez

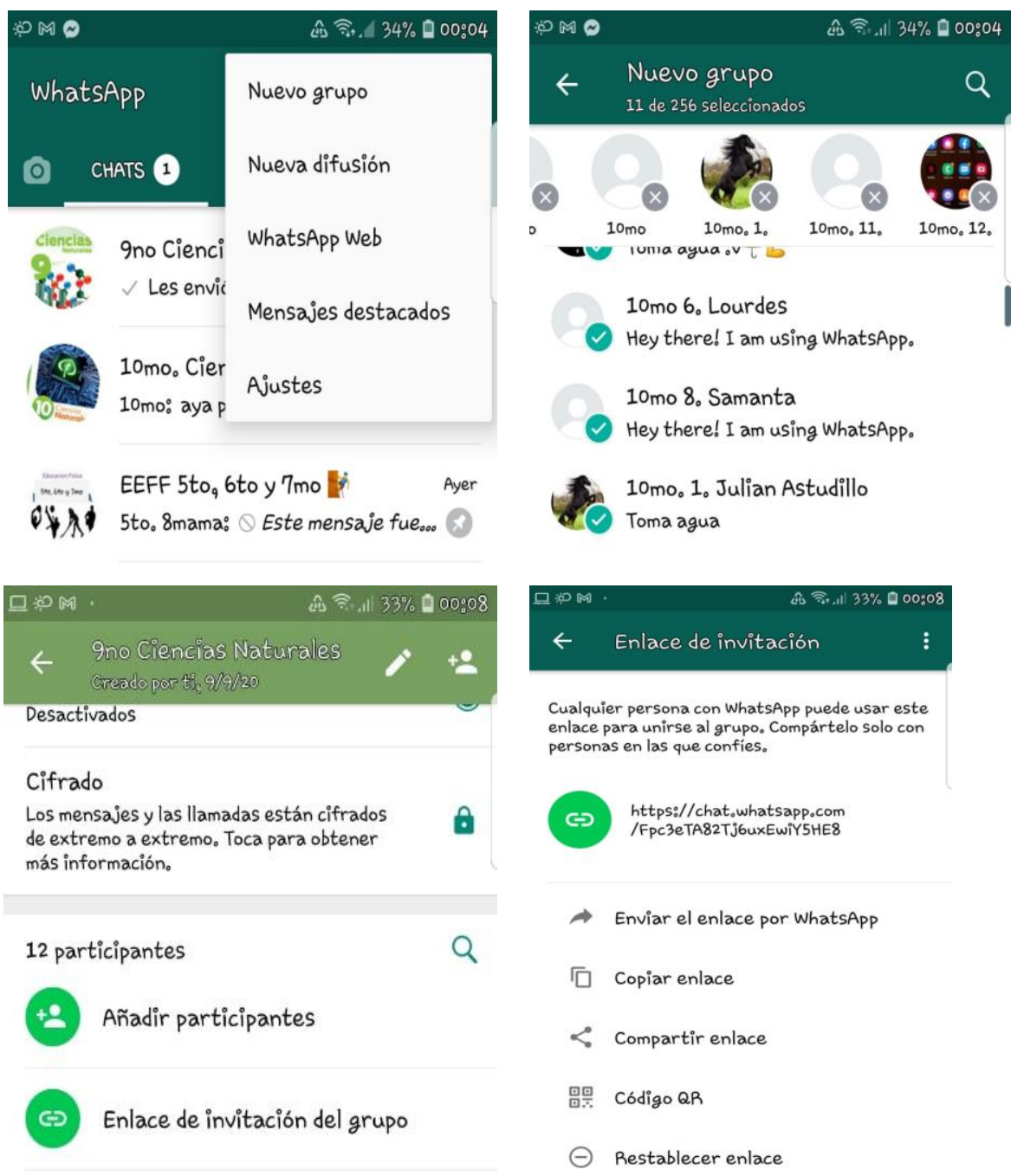

Figura 4. Uso de WhatsApp.

Fuente: Elaboración propia 
Universidad Nacional Experimental Francisco de Miranda (UNEFM). Santa Ana de Coro. Venezuela

Priscila Nataly Chicaiza-Morocho; Darwin Gabriel García-Herrera; Sandra Elizabeth Mena-Clerque Juan Carlos Erazo-Álvarez

Los grupos de trabajo tienen como finalidad dinamizar el aprendizaje y la interacción entre estudiantes y el docente, por lo que se indica las actividades a ejecutar para que dicha finalidad se cumple a cabalidad.

1. Definir el tema de estudio según la asignatura.

2. Asignar roles a los estudiantes.

3. Crear sub grupos de trabajo. (docente-estudiantes)

4. Crear espacios de investigación y retroalimentación dentro del grupo

5. Exposición de trabajos a través de audios y videos.

6. Consultas de inquietudes por medio de llamadas o videollamadas individuales o grupales.

Elaboración de normativas: es fundamental establecer normativas del uso y el comportamiento que se debe tener dentro de los grupos de WhatsApp, pues estas permitirán fomentar ambientes de confianza entre estudiantes y el docente, evitando que se dé el bullying cibernético. Las normativas que pueden establecerse dependiendo del contexto y realidad del grupo, por ejemplo:

1. No emitir comentarios negativos.

2. Reconocer los trabajos de los compañeros con mensajes positivos que realcen su desempeño.

3. Respetar los horarios definidos a través de consenso dentro del grupo, en cuando al intercambio de información, presentación de trabajos, exposiciones, foros, etc.

4. Confirmar la recepción de las notificaciones enviadas por el o la docente.

5. Respetar la temática del grupo creado.

6. Aportar significativa y positivamente en los distintos temas de estudio.

Es importante que las normativas establecidas se encuentren descritas en el apartado de la descripción del grupo, esto ayudará tanto a estudiantes y docentes a mantener una 
Revista Interdisciplinaria de Humanidades, Educación, Ciencia y Tecnología

Año VI. Vol. VI. N³. Edición Especial III. 2020

Hecho el depósito de ley: pp201602FA4721

ISSN-L: 2542-3029; ISSN: 2610-802X

Universidad Nacional Experimental Francisco de Miranda (UNEFM). Santa Ana de Coro. Venezuela

Priscila Nataly Chicaiza-Morocho; Darwin Gabriel García-Herrera; Sandra Elizabeth Mena-Clerque Juan Carlos Erazo-Álvarez

relación cordial y organizada. En la figura 5 y 6 se puede observar al grupo creado y las normas en el WhatsApp.

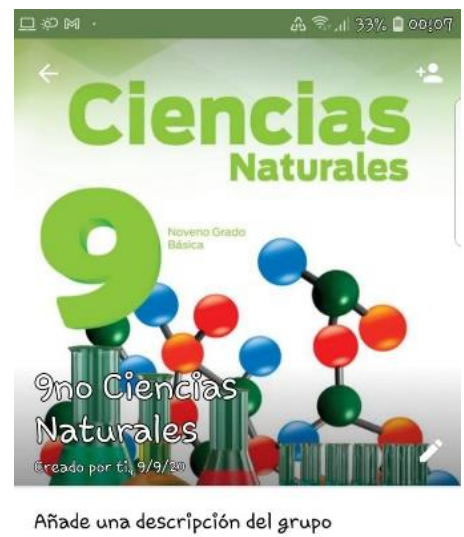

Figura 5. Grupo de WhatsApp creado.

Fuente: Elaboración propia.

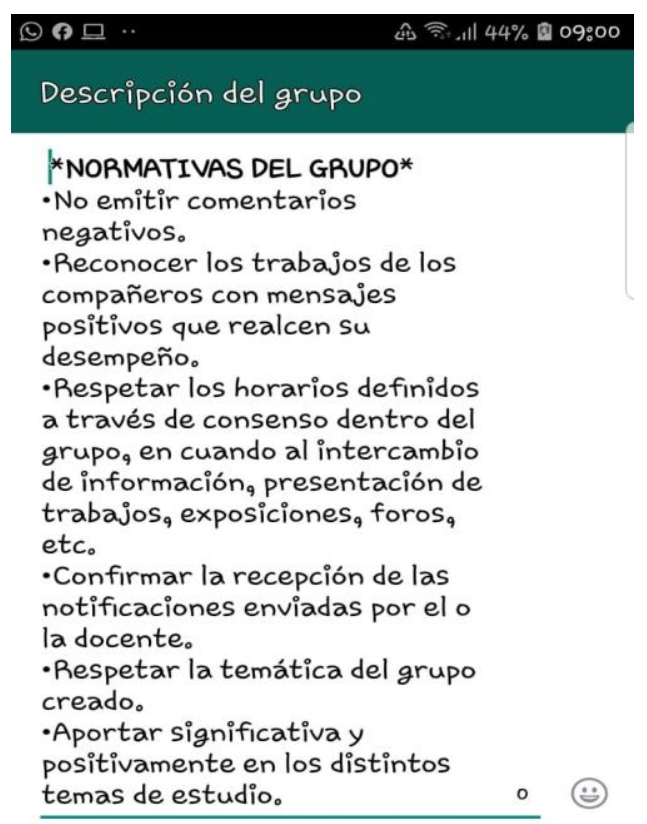

Figura 6. Colocación de normativas en el grupo.

Fuente: Elaboración propia. 
Universidad Nacional Experimental Francisco de Miranda (UNEFM). Santa Ana de Coro. Venezuela

Priscila Nataly Chicaiza-Morocho; Darwin Gabriel García-Herrera; Sandra Elizabeth Mena-Clerque Juan Carlos Erazo-Álvarez

Intercambio de opiniones: otra actividad que se puede realizar en WhatsApp es la publicación de estados (fotos y videos), que son visibles por el lapso de 24 horas para las personas que se encuentren registrados como contacto, la pregunta es ¿cómo esta actividad brinda un aprendizaje en los estudiantes? ¡Muy fácil!, el docente genera un tema de discusión publicando en el estado, el cual los estudiantes tendrán que participar a través de un comentario o argumentando su punto de vista siguiendo las normas establecidas, esto creará un espacio de interacción entre alumnos y el docente. De esta manera todos aprenden de todos, adquiriendo un aprendizaje en conjunto y a su vez una retroalimentación significativa.

\section{CONCLUSIONES}

El WhatsApp se ha convertido en un recurso de aprendizaje que pocos docentes lo utilizan de manera correcta en el ámbito educativo, debido al desconocimiento de sus funciones, utilidades y beneficios, es así que se propuso las tres estrategias mencionadas anteriormente, que permitirán al docente poner en práctica con sus estudiantes.

Los docentes en su mayoría han cambiado su perspectiva con respecto al uso del WhatsApp, debido a que reconocen que puede ser empleado como una estrategia de aprendizaje, especialmente para desarrollar el trabajo colaborativo, pero siendo fundamental definir la edad que deben tener los estudiantes para su uso, es por ello que en la encuesta aplicada, los docentes coinciden que su aplicación se dé con estudiantes de la básica superior, quienes por su edad y autonomía, y con la supervisión constante del docente y de sus representantes, podrán sacar el mayor provecho de esta herramienta tecnológica.

El WhatsApp es un recurso interdisciplinario, ya que puede ser utilizado por todos los docentes de las distintas áreas de estudio, siendo indispensable la creación de normativas para su correcto funcionamiento, fomentando una sana convivencia, mediante espacios de interacción, de esta manera se alcanzará a fortalecer el trabajo en 
Universidad Nacional Experimental Francisco de Miranda (UNEFM). Santa Ana de Coro. Venezuela

Priscila Nataly Chicaiza-Morocho; Darwin Gabriel García-Herrera; Sandra Elizabeth Mena-Clerque Juan Carlos Erazo-Álvarez

conjunto y el desarrollo de emociones positivas, perdiendo el temor a equivocarse y el de opinar libremente.

Cabe destacar, que el presente trabajo, es el resultado de la necesidad que presentan las instituciones educativas de zonas rurales, que ven al WhatsApp, como una herramienta tecnológica accesible, pues por sus condiciones geográficas y socioeconómicas, son factores que les impiden estar a la par de escuelas urbanas, por tal motivo, es urgente que esta App, sea aplicada como una herramienta educativa primordial, dejando de lado estrategias tradicionales y obsoletas, que crean grandes desfases en la formación académica de los estudiantes.

\section{REFERENCIAS}

Benítez , E., \& Marquina , R. (2018). El uso de WhatsApp para el acompañamiento y fomento del trabajo colaborativo en cursos virtuales de Educación Continua. [The use of WhatsApp for the accompaniment and promotion of collaborative word in virtual Continuing Education courses]. Revista de Tecnología de Información y Comunicación en Educación, 12(1), 21-32.

Casillas, S., Cabezas, M., \& Hernández, A. (2017). Construcción de conocimiento colaborativo mediado tecnológicamente: Aportaciones teóricas desde el análisis de prácticas educativas. [Technology-mediated collaborative learning: theoretical contributions based on analysis of educational practice]. Teoría de la Educación. Revista Interuniversitaria, 29(2), 61-86.

Colorado, P., \& Gutiérrez, L. (2016). Estrategias didácticas para la enseñanza de las ciencias naturales en la educación superior. [Didactic strategies for natural sciences teaching in higher education]. Revista Logos, Ciencia \& Tecnología, 8(1), 148-158. Obtenido de https://n9.cl/21k4r

Delgado , M., \& Solano, A. (2009). Estratégias didácticas creativas en entornos virtuales para el aprendizaje. [Creative didactic strategies in virtual surroundings for the learning]. Actualidades Investigativas en Educación, 9(2), 1-21. 
Universidad Nacional Experimental Francisco de Miranda (UNEFM). Santa Ana de Coro. Venezuela

Priscila Nataly Chicaiza-Morocho; Darwin Gabriel García-Herrera; Sandra Elizabeth Mena-Clerque Juan Carlos Erazo-Álvarez

Gómez, M. (2017). Utilización de WhatsApp para la Comunicación en Titulados Superiores. [WhatsApp Use for Communication among Graduates]. REICE. Revista Iberoamericana sobre Calidad, Eficacia y Cambio en Educación, 15(4), 5165. doi:https://doi.org/10.15366/reice2017.15.4.003

Marañón, C. (2012). Redes sociales y jóvenes: Una intimidad cuestionada en internet. [Social and youth media: A questioned privacy on the internet]. Aposta. Revista de Ciencias Sociales(54), 1-16. Obtenido de https://n9.cl/agln7

Quispe, W. (2019). Uso de WhatsApp y su valoración en el trabajo colaborativo de estudiantes de la Maestría en Gerencia de Servicios de Salud de la Facultad de Medicina de la Universidad Nacional Mayor de San Marcos, 2019. [Use of WhatsApp and its assessment in the collabora. Universidad Nacional Mayor de San Marcos. Recuperado de https://n9.cl/y7zy

Rodríguez, D. (2020). Más allá de la mensajería instantánea: WhatsApp como una herramienta de mediación y apoyo en la enseñanza de la Bibliotecología. [Beyond instant messaging: WhatsApp as a mediation and support tool in the teaching of Library Science]. Información, Cultura y Sociedad, (42), 107-126. doi:https://doi.org/10.34096/ics.i42.7391

Rubio, J., \& Perlado, M. (2015). El fenómeno WhatsApp en el contexto de la comunicación personal: una aproximación a través de los jóvenes universitarios. [The WhatsApp phenomenon in the context of personal communication: an approximation through the university youths]. Revista ICONO14 Revista Científica De Comunicación Y Tecnologías Emergentes, 13(2), 73-94. doi:https://doi.org/10.7195/ri14.v13i2.818

Ruvalcaba, L., Torres, V., Carmona, E., \& Pérez, Ó. (2019). Perfil estudiantil: uso de WhatsApp y Facebook. [Student profile: use of Whatsapp and Facebook]. NOVUM, revista de Ciencias Sociales Apliacadas, 1(9), 32-57. Obtenido de https://n9.cl/3f12o

Siemens, G. (2004). Elearnspace. Connectivism: A Learning Theory for the Digital Age. Recuperado de https://n9.cl/5jw3i 
Universidad Nacional Experimental Francisco de Miranda (UNEFM). Santa Ana de Coro. Venezuela

Priscila Nataly Chicaiza-Morocho; Darwin Gabriel García-Herrera; Sandra Elizabeth Mena-Clerque Juan Carlos Erazo-Álvarez

Suárez, B. (2017). WhatsApp como herramienta de apoyo a la tutoría. [WhatsApp as support tool in tutoring]. Revista de Docencia Universitaria, 15(2), 193-210. Obtenido de https://n9.cl/8i9d4

Tezer, M., Taşpolat, A., Sami , K., \& Fatih , S. (2017). The impact of using social media on academic. [El impacto del uso de las redes sociales en el académico]. International Journal of Cognitive Research in Science, Engineering and Education, 5(2), 75-81. doi:https://doi.org/10.5937/ijcrsee1702075T

Vilches, M. (2019). Utilización de WhatsApp para el trabajo grupal por el alumnado de los títulos de grado en educación de la Universidad de Córdoba. [Use of WhatsApp for group work by students of undergraduate degrees in education at the University of Córdoba]. Recuperado de https://n9.cl/yso3

Zulkanain, N., Miskon, S., \& Syed, N. (2020). An adapted pedagogical framework in utilizing WhatsApp for learning purpose. [Un marco pedagógico adaptado para utilizar WhatsApp con fines de aprendizaje]. Education and Information Technologies, 25, 2811-2822. doi:https://doiorg.vpn.ucacue.edu.ec/10.1007/s10639-019-10096-0

C2020 por los autores. Este artículo es de acceso abierto y distribuido según los términos y condiciones de la licencia Creative Commons Atribución-NoComercial-Compartirlgual 4.0 Internacional (CC BY-NC-SA 4.0) (https://creativecommons.org/licenses/by-nc-sa/4.0/) 